\title{
Perspective
}

\section{What If Quantum Computer Combined with Artificial Intelligence?}

Mohammad Ranjan, M.Sc.; Hansen Hopper, M.Sc.

\begin{abstract}
With the rapid progress in computation, our ability to deal with mega data is enhanced dramatically. Quantum computer is the latest development that promises us with great contribution to the upcoming generation of artificial intelligence. The positive feedback cycle formed between quantum computer and artificial intelligence is the most eye-catching point that possesses the potential to reach the goal of self-learning and self-improvement. The new era of technology has approached, the only thing we need to do is to enhance and upgrade our knowledge to prevent being knocked out.
\end{abstract}

KEYWORDS Quantum computer; Artificial intelligence; Human workforce; Military; Future generation

Sci Insigt. 2019; 29(2):48-51. doi:10.15354/si.19.pe026
Author Affiliations: Author affiliations are listed at the end of this article.

Correspondence to:

Mr. Hansen Hopper, M.Sc., Division of Physics, The BASE, Chapel Hill, NC 27510, USA

Email: han-

sen.hopper@basehq.org

Copyright (C 2019 Insights Publisher. This is an open access article distributed under the Creative Commons Attribution License, which permits unrestricted use, distribution, and reproduction in any medium, provided the original work is properly cited. 
$\mathrm{E}$ ARLY this year, on January 8th, 2019, IBM unveiled IBM Q System One ${ }^{\mathrm{TM}}$, the world's first integrated universal approximate quantum computing system designed for scientific and commercial use at the 2019 Consumer Electronics Show (CES), and IBM also announced plans to open its first IBM Q Quantum Computation Center for commercial clients in Poughkeepsie, New York in 2019 (1). This is a huge step. Quantum computers, which used to be out of reach, suddenly approached us.

How strong is a quantum computer? Let's just say that a desktop computer-sized quantum computer can reach or exceed the computing power of today's most advanced Chinese Tianhe-1 supercomputer (2). For example, to solve a commonly used Rivest-Shamir-Adleman (RSA) cryptosystem, it takes 600,000 years to use the current largest and best supercomputer, but with a quantum computer with considerable storage function, it takes less than 3 hours (3). In the face of quantum computers, the traditional electronic computers we once proud of are equivalent to ancient abacus, which was cumbersome and old. The world will experience a subversive change, regardless of production, research or daily life.

Why are quantum computers so powerful? From the point of mechanism, quantum computers and traditional computers are absolutely different in their working principle and path. In an existing computing unit of a traditional electronic computer, one bit has only a particular state at a specific time, either 0 or 1 . However, for quantum computers, a quantum-specified "superposition state" is used to take parallel computations, and the ultimate goal is to make the computation speed increase exponentially (4). Let's have a simple instance, it is pretty hard for human beings to choose the direction when they were facing several directions under an emergency and confusing situation because they do not know which way was correct. Similarly, traditional computers to solve the problem are also this kind of fate: they can only take one route first, and then take route road, that means they cannot walk two routes together. Nevertheless, quantum computers are not the same. It can be like parallel computing in the case that one big computer has changed to many small computers to take different paths to solve arithmetic problems by millions of calculators at the same time. The huge leap from traditional electronic computers to quantum computers, the entire computing power and the ability to process mega data of human being will increase by thousands or even hundreds of millions times.

This is really a perfect time for the upcoming IBM Quantum Computer: 5G network is mature and close to use, big data is booming, and artificial intelligence has come to the door. In this way, we cannot imagine what the consequence would be if we combine the quantum computer with the artificial intelligence. Quickly, the "cold-blooded" quantum computers will have the ability to self-learn and think like a human being, and in turn, will overcome us human being. This sounds like science fiction, but it is really the fact need to be considered seriously. As the quantum computing plus artificial intelligence is approaching in a much faster speed than the most radical imagination we have ever had. The major reason is both will realize positive feedback, i.e., quantum computer plus artificial intelligence will continue to iterate out more advanced combination of quantum computers and artificial intelligence, and then make the slope of development steep suddenly in an unimaginable speed.

A little bit earlier, The Defense Advanced Research Projects Agency, part of the Defense Department, said it will invest up to $\$ 2$ billion over the next five years toward new programs advancing artificial intelligence (5). This seemingly inconspicuous news has actually made many people scare a cold sweat. Have you remembered the horrible video that was made public at a meeting of the United Nations Weapons Convention in Geneva, attended by more than 70 countries in 2018 ? In the video, a terror weapon the killer robot was exposed. This killer robot is actually a small intelligent drone, just as big as a bee, but its processor is 100 times faster than humans, and it can avoid human tracking. Although the bees were small, they had complete implantation of the latest technologies of the whole body: wide-angle camera, sensor, facial recognition and so forth. As long as the target image information is input into it, it will accurately find the target with a recognition rate as high as 99.99 , that means it is useless of wearing masks and camouflage. In addition, each killer robot is equipped with 3 grams of concentrated explosives. Once the target is determined, it can reach headshot without any pressure by destroying the entire brain. And it can penetrate buildings, cars, and trains, and avoid human bullets, and deal with almost all kinds of defenses.

If a homicide robot that costs only \$25 million was released, it will kill people in half a city. The only thing you need to do is to pick out the enemy and define each person's facial information, the bee colony will then launch a targeted attack. So now, the US military has actually begun to study artificial intelligence projects, which are likely to include such artificial intelligent killers. It is conceivable that once this technology was widely used, it will escalate the conflict of war to an unprecedented scale, and it will be difficult for humans to control the situation. What is even more frightening is that if a selfish scientist adds a line of instructions to destroy humans in the code, or artificial intelligence suddenly mutates into anti-human species, the whole human being will be swept by the robot. That was why, in an interview, Elon Musk, CEO of SpaceX, stated that we have only $5 \%$ to $10 \%$ probability of ensuring the safety of artificial intelligence. "We need to be extremely vigilant about artificial intelligence, and they are more dangerous than nuclear weapons." Musk continued. That is also the reason why Stephen Hawking, a theoretical physicist at the University of Cambridge, warned humans that 
robots may evolve faster than we can imagine, and their ultimate goal will be unpredictable.

Today, the future has come, and we have reached the door of destiny. Yes, from today, quantum computer plus artificial intelligence, it is no longer a science fiction, no longer a reading comprehension, no longer a news headline, no longer a byte in Ethernet and an inconspicuous "soul" in the CPU, but a real fate. In the era of quantum computer plus artificial intelligence, this, for our human society, is a tsunami full of uncertainty. The biggest feature of this revolution is that it does not change what we do, but it changes ourselves. If the previous technological revolutions were at most the extension and replacement of human organs, such hands and feet, this time, quantum computer plus artificial intelligence will become a substitute for human beings. Its impact on the human society and the society as a whole will be unprecedented.

The most direct impact of the combination of both quantum computer and artificial intelligence would be as it is rapidly surpassing humans. This also means that a large number of translators, journalists, cashiers, assistants, secu- rity guards, drivers, traders, customer service and so on may lose their jobs in the near future. In this regard, Jerry Kaplan, Professor of Artificial Intelligence and Ethics from Stanford University had made estimation that $47 \%$ of the 720 types of jobs registered in the US will be replaced by artificial intelligence, and in China, this ratio would exceed $70 \%$. As one part of the historical process, the most important task of life is to "follow the direction of historical operation", and not to be inexplicably crushed by history. In the face of artificial intelligence, we cannot change the process of technology, but we can change ourselves and the knowledge structure of our next generations.

When facing the combination of both quantum computer and artificial intelligence, we have only three choices can do: either accumulate wealth and become a capital predator, or accumulate fame and become a unique individual, or accumulate knowledge and become the master of advanced technology. The new era has gradually approached, and we must consciously upgrade ourselves in order to catch up with the trend of the times.

\section{ARTICLE INFORMATION}

Author Affiliations: CyberWalk Co., New Delhi 110055, India (Ranjan). Division of Physics, The BASE, Chapel Hill, NC 27510, USA (Hopper).

Author Contributions: Hopper had full access to all of the data in the study and takes responsibility for the integrity of the data and the accuracy of the data analysis.

Study concept and design: Ranjan, Hopper. Acquisition, analysis, or interpretation of data: Ranjan.

Drafting of the manuscript: Ranjan, Hopper.
Critical revision of the manuscript for important intellectual content: Hopper. Statistical analysis: $\mathrm{n} / \mathrm{a}$.

Obtained funding: $\mathrm{n} / \mathrm{a}$.

Administrative, technical, or material support: Hopper.

Study supervision: Hopper.

Conflict of Interest Disclosures: The author declared no competing interests of this manuscript submitted for publication.

Acknowledgement: $n / a$.

Funding/Support: $n / a$
Role of the Funder/Sponsor: $n / a$.

How to Cite This Paper: Ranjan M, Hopper H. What if quantum computer combined with artificial intelligence? Sci Insigt 2019; 29(2): 48-51.

Digital Object Identifier (DOI): http://dx.doi.org/10.15354/ si.19.pe026.

Article Submission Information: Received, March 26, 2019; Revised: May 06, 2019; Accepted: May 08, 2019..

\section{REFERENCES}

1. Nay C. IBM unveils world's first integrated quantum computing system for commercial use. Last Accessed: July 4th, 2019. https://newsroom.ibm.com/2019-0108-IBM-Unveils-Worlds-FirstIntegrated-Quantum-ComputingSystem-for-Commercial-Use

2. Sayer P. IBM ends China's 5-year reign atop supercomputer rankings.
Last Accessed: July 4th, 2019. https://www.networkworld.com/article /3284066/ibm-system-ends-chinasfive-year-reign-at-top-ofsupercomputer-rankings.html

3. Simmons GJ. RSA encryption. Last Accessed: July 4th, 2019. https://www.britannica.com/topic/RS A-encryption
4. Medhane DV, Sangaiah AK. Chapter 13 - A quantum-inspired hybrid intelligent position monitoring system in wireless networks. Quantum Inspired Computational Intelligence 2017; pp417-pp452.

5. Harwell D. Defense Department pledges billions toward artificial intelligence research. Last Accessed: Ju- 
ly 4th, 2019.

https://www.washingtonpost.com/tec hnology/2018/09/07/defense- department-pledges-billions-towardartificial-intelligence- re-

search/?utm_term=.8168662f5239 